\title{
Grátis não é o suficiente: estudo da adoção individual da telefonia pela internet
}

\section{Free is not enough: study of the individual adoption of Internet telephony}

\author{
ALEXANDRE CAPPELLOZZA* \\ OTÁVIO PRÓSPERO SANCHEZ**
}

\section{RESUMO}

Estudos têm demonstrado a existência de diversos fatores de influência da adoção de Tecnologias da Informação. Para a operacionalização de uma análise de adoção de tecnologia que englobe múltiplas interações de influências, optou-se pela contextualização do estudo com foco na telefonia pela internet, pois quando comparada com outras tecnologias de comunicação, a gratuidade de ligações é um dos principais benefícios, aliada a outros recursos. A pesquisa fundamenta-se em uma abordagem quantitativa, onde os dados foram obtidos com a participação de 313 usuários de telefonia pela internet. Realizou-se análise de equações estruturais para confirmar relações entre os fatores. Os resultados indicam que o hábito influencia mais o uso gratuito dessa tecnologia do que a percepção dos benefícios do uso em si, apesar da ausência de custos das ligações ser um dos principais incentivos à adoção da tecnologia. Evidencia-se que a rede de contatos do usuário é o principal responsável pela formação do hábito de uso das tecnologias de comunicação. A disponibilidade também mostrou-se um fator à formação do hábito. Sob o ponto

* Universidade Metodista de São Paulo. Professor Titular do Programa de Pós-Graduação em Administração da Universidade Metodista de São Paulo.

alexandre.cappellozza@metodista.br .

** Otávio Próspero Sanchez. Escola de Administração de Empresas de São Paulo - FGV. otavio.sanchez@fgv.br . 
de vista dos interessados em fomentar o uso de tecnologias inovadoras de comunicação, este estudo contribui na exposição de fatores que promovem a adoção desses sistemas.

Palavras-chave: Tecnologia. Adoção. Internet.

\section{Abstract}

Studies have shown the existence of several factors that influence the adoption of communication technologies. In order to perform an empirical analysis covered of multiple influences, we chose to focus on Internet Telephony, as compared to other medias. Its gratuitousness is one of the key benefits, besides other resources available to the users. The research is based on a quantitative approach, where the data was obtained with the participation of 313 users. Structural equations modelling were performed to confirm relationships between the factors. Our results indicate that habit is the main influence towards the use of this technology, despite the toll free calls. The user's social network is a key driver for the formation of the habit of use of VOIP technology. Also, the accessibility posits itself as another positive adoption factor. From the point of view of those interested in promoting the use of innovative communication technologies, this study contributes to the exposure of drivers that promotes the adoption of these systems.

Key-words: Technology. Adoption. Internet.

\section{INTRODUÇÃo}

Entre diversas formas de comunicação digital, a tecnologia de Voz sobre Protocolo de Internet - VoIP - oferece benefícios multifacetados, pela possibilidade de suportar a comunicação por voz sem custo tarifário, além de métodos variados de troca de informações entre destinatários, por exemplo as mensagens instantâneas, imagens ou arquivos (PESSEMIER et al., 2016).

Dada sua capacidade de suporte à comunicação, a telefonia VoIP deveria ser a opção preferida dos usuários para comunicação. No entanto, apesar de diversos sistemas VoIP estarem acessíveis há algum tempo, pesquisas indicam que o número de usuários de telefonia VoIP, embora crescente nos últimos anos, não supera o número de usuários das tecnologias de telefonias tradicionais, 
além de não se posicionar como o serviço preferido de comunicação pessoal (IDG, 2011).

A adoção de tecnologias é tratada na literatura sobre várias perspectivas. Por exemplo, a adoção pode ocorrer em ondas em que indivíduos afeitos a novidades tendem a experimentar antecipadamente, seguidos pela maior quantidade de usuários, que esperam que os benefícios sejam claramente identificados e os riscos minimizados (ROGERS, 1995), como resultados de uma análise objetiva de benefícios como utilidade e facilidade de uso (DAVIS, 1989). Mais recentemente, pesquisas têm evoluído na direção de analisar como o hábito de utilizar uma tecnologia existente influi na resistência de adoção de uma nova tecnologia (VENKATESH et al., 2012). Entretanto, embora possa também apresentar influência sobre o emprego da nova tecnologia, os antecedentes na formação do hábito de usar uma nova tecnologia permanecem pouco explicados pela literatura (POLITES; KARAHANNA, 2012).

Identificar os elementos que compõem o hábito de uso individual de novas tecnologias é importante, porque permite compreender como se estabelecem usos sustentados de uma nova tecnologia, e permite melhor compreensão sobre a velocidade esperada de adoção de tecnologias.

Este estudo analisa como o hábito de uso da tecnologia de comunicação de VoIP é formado, e como o hábito de uso é associado ao seu uso. A pesquisa, conduzida por meio de survey com estudantes, indica que, contrariamente ao esperado, o hábito de uso de telefonia VoIP, em média, não se forma predominantemente por uma percepção de custos baixos, mas a compatibilidade de acesso à rede de contatos é considerada o fator mais relevante. A baixa padronização entre os usuários de telefones móveis e de aplicativos de acesso à telefonia VoIP pode explicar por que a telefonia VoIP, embora virtualmente grátis, não teve uma adoção radicalmente elevada até o momento. 


\section{REFERENCIAL TEÓRICO}

\subsection{A telefonia pela internet}

A criação de novos e melhores componentes eletrônicos permitiu o desenvolvimento das telecomunicações, computadores e sistemas de informação, o que possibilitou uma conexão mundial das redes de computadores e telefonia para tornar possível a convergência na utilização desses equipamentos. A telefonia via internet, também conhecida como telefonia IP ou Tecnologia VoIP emergiu em 1995, quando uma companhia de software chamada Vocaltec desenvolveu um programa de comunicação VoIP (CECERE, 2009).

Em constante desenvolvimento e crescente volume de adoção desde então, a presença da tecnologia VoIP pode ser observada pela sua utilização em diferentes plataformas de comunicações, como: smartphones, computadores pessoais, aparelhos telefônicos híbridos que mesclam diferentes tipos de tecnologia de telefonia, interfaces corporativas para redução de custos de ligações e sistemas de comunicação empresariais (SHIN, 2012).

Adiciona-se que os sistemas VoIP podem proporcionar maior mobilidade dos usuários, além de flexibilizar e diversificar os meios de comunicação interpessoal: alguns sistemas VoIP conseguem estabelecer conferências telefônicas, videochamadas, gravação de recados, entre outros recursos. A inclusão de funcionalidades aos sistemas VoIP é um aspecto que pode favorecer a adoção dessas tecnologias entre os usuários desses sistemas (TOBIN; BIDOLI, 2006): a gama de funcionalidades contidas no sistema VoIP pode proporcionar diversos métodos de transmissão de informações aos destinatários por meio do envio de mensagens instantâneas, imagens ou arquivos (PESSEMIER et al., 2016).

Observa-se que as operadoras tradicionais possuem modelos de negócio tradicionais ancorados na geração compulsória de receitas pelos seus clientes e, por vezes, aprisionando-os contratualmente (DE BIJL; PEITZ, 2009), por sua vez, a telefonia pela internet exibe características de tecnologias disruptivas, pois altera o modelo de negócio de comunicação telefônica anulando, praticamente, os custos marginais sobre ligações telefônicas na sua rede de usuários e isentando o usuário de armadilhas publicitárias que obrigam o 
usuário a utilizar tecnologias indesejadas. Para se compreender como diferentes aspectos podem envolver a decisão de uso de uma tecnologia de informação, recorre-se aos estudos de adoção desses recursos. Ao longo dos últimos anos, a ubiquidade e a diversidade das plataformas digitais de informação e comunicação disponíveis aos usuários possibilitam estudos que versam sobre o estudo de modelos de adoção de tecnologias que atendam tanto a necessidades profissionais, quanto pessoais (KWAK; LEE, 2012).

\subsection{A Adoção de Tecnologias de Informação}

A literatura de adoção de tecnologias de Informação expõe múltiplas análises e estudos das diferentes razões que favorecem o uso de tecnologias de informação e comunicação (VENKATESH et al., 2012).

O desenvolvimento de modelos à adoção de TI possibilita previsões sobre os comportamentos dos usuários com a oferta de tecnologias ou sistemas; de modo que diversos construtos sobre a adoção de TI podem auxiliar a compreensão acerca do comportamento de uso das aplicações. Nessa direção, diversas influências podem alterar as intenções de uso dos decisores sobre as suas escolhas a respeito de tecnologias de informação, sejam essas influências guiadas, por exemplo, por características individuais, disponibilidade de recursos, e aspectos sociais, como: hábito, disponibilidade da aplicação e rede social dos contatos do usuário, respectivamente (KERAMATI et al., 2012; POLITES, 2012).

\section{Hábito}

O hábito pode ser definido como a tendência comportamental das pessoas de fornecerem, automaticamente, respostas idênticas, desde que suportadas em um contexto situacional, outrora, semelhante (LIMAYEM et al., 2007).

A influência do hábito sobre o comportamento pode ser explicada por meio de uma ligação cognitiva entre uma situação e uma respectiva ação e essa ligação cognitiva é formada a partir dos resultados anteriores dessa mesma ação em outras situações idênticas (KIM; MALHOTRA, 2005). Por exemplo, a necessidade de comunica- 
ção aliada à memória da imagem de uma conversação por telefone pode iniciar o processo cognitivo que resultará na ligação telefônica do usuário a um terceiro e assim, habitualmente, colaborar para o uso dessa tecnologia de comunicação (GUINEA; MARKUS, 2009; KERAMATI et al., 2012). Portanto, elabora-se a seguinte hipótese:

H1: O hábito influencia positivamente o comportamento de uso da telefonia pela internet

\section{Utilidade Percebida}

Entre os diversos modelos que objetivam o estudo da adoção de TI, o modelo TAM é o mais empregado para pesquisas de aceitação e uso de tecnologias (PLOUFFE et al., 2001). Entre os construtos componentes desse modelo, a utilidade percebida caracteriza-se pela maior relevância na avaliação da aceitação de tecnologias de informação pelo efeito direto na intenção e uso da TI. A Utilidade Percebida é definida como o "grau em que uma pessoa acredita que utilizar um determinado sistema melhorará seu desempenho em sua tarefa/trabalho" (DAVIS et al., 1989). Assim, formula-se a segunda hipótese deste estudo:

H2: A utilidade percebida influencia positivamente o comportamento de uso da telefonia pela internet

Sabe-se que a utilidade percebida é formada a partir de um processo cognitivo individual a respeito das vantagens obtidas pelo uso da aplicação tecnológica (DAVIS et al., 1989; BROWN et al., 2014). Já o uso contínuo, ou habitual, de TI é influenciado por um conjunto de decisões racionais que podem incluir diferentes percepções sobre a aplicação como experiências positivas anteriores com o recurso tecnológico (BHATTACHERJEE, 2001). Dessa forma, elabora-se a seguinte hipótese:

H3: A utilidade percebida influencia positivamente o hábito de uso da telefonia pela internet

\section{Disponibilidade}

Entende-se a disponibilidade de uso de determinada tecnologia como uma percepção que envolve a conveniência e acessibilidade 
percebidas ao uso desse recurso. A conveniência pode ser definida como a possibilidade de um indivíduo receber um serviço quando desejar. Os indivíduos costumam valorizar a conveniência dos serviços pela forma, período e tempo para obtenção do desempenho desejado (HU et al., 2009).

Pesquisas demonstram a influência da percepção de conveniência, ou conveniência percebida, ao uso de diversas aplicações de TI, como: tecnologias Wireless, compras via internet (GUPTA; KIM, 2010), uso de sistema de mensagens eletrônicas por meio de telefone celular (KIM et al., 2008).

Pressupõe-se que a escolha da tecnologia que fornece maior conforto ao uso da TI perpassa pelo livre acesso dos recursos computacionais a fim de permitir o uso a qualquer momento ou local (PESSEMIER et al., 2016). Dessa forma, a percepção de conveniência sobre o uso da tecnologia pode promover uma valorização positiva da utilidade percebida da TI ao usuário (KIM et al., 2008; KIM, 2009) e influenciar o hábito de uso da aplicação. Assim, levantam-se duas hipóteses acerca dos efeitos da disponibilidade à adoção da tecnologia VoIP:

H4: A disponibilidade influencia positivamente a utilidade percebida da telefonia pela internet

H5: A disponibilidade influencia positivamente o hábito de uso da telefonia pela internet

\section{Rede Social}

De acordo com Markus (1987), após determinada proporção de usuários ser atraída pela tecnologia, ou massa crítica, o uso é disseminado entre a comunidade desses usuários de forma rápida.

Assim, a massa crítica se trata de um momento que o uso da inovação está, suficientemente, difundido e se torna sustentável (VAN SLYKE et al., 2007). Portanto, uma das hipóteses de explicação sobre a adoção expressiva de uma inovação pode se referir ao alcance de massa crítica de usuários que utilizam determinada tecnologia (GLASS; LI, 2010).

Em um contexto de tecnologia de comunicação, caso o usuário note que seus contatos não estão disponíveis, ou desconectados, da 
tecnologia em questão, o usuário pode evitar o uso dessa tecnologia por entender que não conseguirá estabelecer conversação efetiva com esses contatos e reduzir a percepção de utilidade (MAINES et al., 2006), assim como dificultar a formação do hábito de uso dessa aplicação. Dessa forma, geram-se as seguintes hipóteses:

H6: A rede social influencia positivamente a utilidade percebida da telefonia pela internet

H7: A rede social influencia positivamente o hábito de uso da telefonia pela internet

\section{Custos}

Entre os diversos aspectos que podem ser notados pelo usuário à adoção da tecnologia VoIP, o estudo dos custos percebidos ao uso de tecnologias de comunicação é um aspecto relevante para aplicações de comunicações de voz, uma vez que as tarifas tendem a ser nulas ou próximas dos custos marginais pela intensa competição no setor de telefonia (KWAK; LEE, 2012).

Além disso, um dos principais benefícios do uso da tecnologia VoIP é a possibilidade de se comunicar por voz sem custo tarifário, o que poderia reduzir os custos individuais com ligações telefônicas (DANTU et al., 2009).

Infere-se que os custos mensais das tarifas cobradas por operadoras de telecomunicações podem gerar uma barreira para contratação de tecnologias de comunicação e, assim, favorecer a adoção de tecnologias que promovam a redução dos custos do usuário e aumentar a percepção de utilidade percebida, bem como, favorecer a utilização habitual da telefonia pela internet (CHUAN-FONG SHIH; VENKATESH, 2004). Portanto, elaboram-se as seguintes hipóteses:

H8: Os custos percebidos influenciam positivamente a utilidade percebida da telefonia pela internet

H9: Os custos percebidos influenciam positivamente o hábito de uso da telefonia pela internet

Elaboradas as hipóteses de pesquisa a serem testadas empiricamente, esquematiza-se o modelo conceitual de pesquisa. 
Figura 1: Modelo Conceitual de Pesquisa

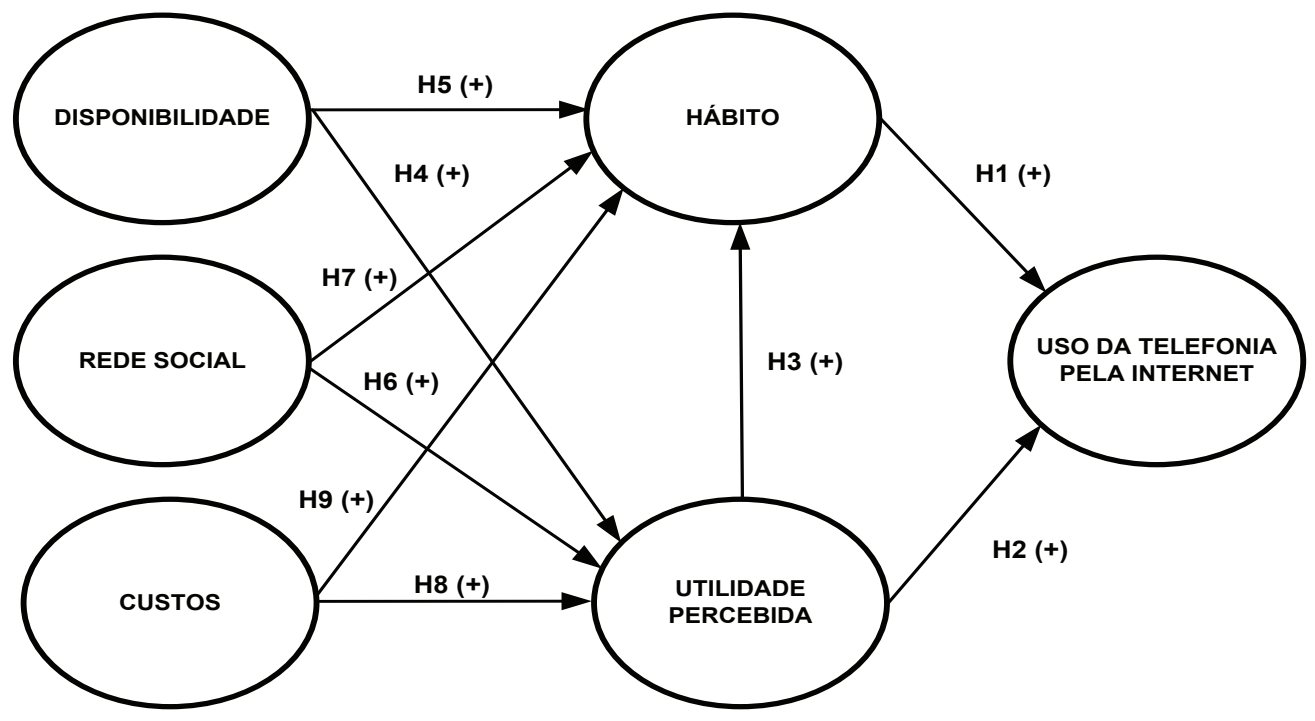

\section{Procedimentos Metodológicos}

\subsection{Informações da amostra e instrumento de medida}

Informa-se que esta pesquisa de corte transversal único será realizada por meio de survey, sob caráter confirmatório e analisará os dados observados de um questionário elaborado para a coleta dos dados empíricos.

Neste estudo, a escala de Likert foi considerada como escala intervalar como uma forma de evitar outliers multivariados, pela sua praticidade de uso, além da possibilidade de uso de afirmações que não estão explicitamente ligadas ao objeto estudado com o objetivo de coleta de informações sobre as percepções individuais dos respondentes (HAIR JR. et al., 2005).

As escalas necessárias para a coleta de dados do construto Utilidade Percebida foram conseguidas de Davis (1989). As escalas de rede social, a disponibilidade, o hábito e os custos percebidos foram obtidos de Xin et al. (2010), Hu et al. (2009) e Gupta e Kim (2010), Limayen et al. (2007), Venkatesh (2003) e Brown e Venkatesh (2005) respectivamente. 
Antes de sua aplicação, o questionário proposto passou por um processo de validação de face por um grupo de cinco pesquisadores informados sobre o tema. Os comentários dos pesquisadores foram analisados e algumas alterações foram executadas no instrumento de medida.

Para a aplicação final do instrumento de medida deste estudo, participaram 313 usuários de sistemas de telefonia pela internet em São Paulo.

Esse número de respondentes supera o número mínimo de respondentes necessários para se alcançar um poder de explicação do teste de 80\% (COHEN, 1977; HAIR JR. et al., 2005).

A idade média dos respondentes é igual a 24,08 anos, com desvio padrão igual a 6,94, o que sinaliza que a amostra é composta de jovens adultos. Também não foi constatada uma hegemonia de gênero na amostra, pois a amostra era composta de $60 \%$ de respondentes femininas.

A localização geográfica para busca dos respondentes da pesquisa foi ponderada: o Estado de São Paulo concentra o maior número de linhas móveis comparado com outros Estados e contempla, aproximadamente, $25 \%$ da quantidade de linhas telefônicas móveis brasileiras (TELECO, 2012).

\subsection{Avaliação do modelo de mensuração e estrutural}

Para realizar o teste das hipóteses deste estudo, serão utilizados testes estatísticos com a técnica de Modelos de Equações Estruturais - MEE pela técnica PLS-PM (Partial Least Squares - Path Modeling).

Acrescenta-se que a utilização do PLS neste estudo também se justifica pelas características deste estudo, dado que é considerado o método mais adequado cujos dados são menos suscetíveis a desvios da normalidade multivariada (LOHMOLLER, 1989; VINZI et al., 2010). Para a realização dos cálculos necessários para a validação do modelo estrutural deste estudo, utilizou-se o software SmartPLS, versão 2.0M3 (RINGLE et al., 2005).

Com os dados coletados do questionário, procedeu-se à avaliação do modelo de mensuração. Todos os resultados apresentados neste tópico estão padronizados. 
Para a validação convergente, recomenda-se que os valores das cargas fatoriais sejam superiores a 0,70 quando pertencentes aos seus respectivos construtos, e para a validação discriminante os valores das cargas fatoriais sejam inferiores quando associados aos outros construtos adjacentes conforme os resultados apresentados na Tabela 1 (CHIN, 2010).

Tabela 1: Cargas Fatoriais dos Indicadores

\begin{tabular}{llccccc}
\hline Variável & Cus tos & Dis ponibilidade & Hábito & $\begin{array}{c}\text { Rede } \\
\text { Social }\end{array}$ & $\begin{array}{c}\text { Utilidade } \\
\text { Percebida }\end{array}$ & $\begin{array}{c}\text { Uso de } \\
\text { Telefonia IP }\end{array}$ \\
\hline CUST1 & $\mathbf{0 , 8 4}$ & 0,47 & 0,51 & 0,35 & 0,43 & 0,40 \\
CUST2 & $\mathbf{0 , 8 9}$ & 0,35 & 0,50 & 0,34 & 0,61 & 0,46 \\
DISP1 & 0,30 & $\mathbf{0 , 8 3}$ & 0,44 & 0,40 & 0,32 & 0,41 \\
DISP2 & 0,31 & $\mathbf{0 , 6 2}$ & 0,32 & 0,30 & 0,29 & 0,33 \\
DISP3 & 0,43 & $\mathbf{0 , 7 7}$ & 0,50 & 0,51 & 0,38 & 0,50 \\
DISP4 & 0,28 & $\mathbf{0 , 7 7}$ & 0,35 & 0,34 & 0,30 & 0,32 \\
DISP5 & 0,45 & $\mathbf{0 , 8 2}$ & 0,53 & 0,46 & 0,39 & 0,49 \\
HAB1 & 0,54 & 0,52 & $\mathbf{0 , 8 8}$ & 0,62 & 0,58 & 0,70 \\
HAB2 & 0,40 & 0,51 & $\mathbf{0 , 8 6}$ & 0,57 & 0,51 & 0,62 \\
HAB3 & 0,57 & 0,46 & $\mathbf{0 , 8 6}$ & 0,49 & 0,52 & 0,58 \\
RED1 & 0,20 & 0,41 & 0,46 & $\mathbf{0 , 7 9}$ & 0,32 & 0,45 \\
RED2 & 0,42 & 0,48 & 0,63 & $\mathbf{0 , 8 6}$ & 0,52 & 0,63 \\
RED3 & 0,32 & 0,43 & 0,49 & $\mathbf{0 , 8 5}$ & 0,37 & 0,46 \\
UTIL1 & 0,54 & 0,34 & 0,58 & 0,39 & $\mathbf{0 , 8 3}$ & 0,47 \\
UTIL2 & 0,49 & 0,36 & 0,46 & 0,42 & $\mathbf{0 , 8 0}$ & 0,38 \\
UTIL3 & 0,47 & 0,40 & 0,48 & 0,43 & $\mathbf{0 , 8 3}$ & 0,41 \\
USO1 & 0,46 & 0,54 & 0,64 & 0,66 & 0,47 & $\mathbf{0 , 8 4}$ \\
USO2 & 0,44 & 0,46 & 0,65 & 0,51 & 0,46 & $\mathbf{0 , 8 9}$ \\
USO3 & 0,38 & 0,42 & 0,59 & 0,46 & 0,41 & $\mathbf{0 , 8 6}$ \\
\hline
\end{tabular}

Para a validação convergente do modelo, também analisam-se os valores da variância média extraída dos construtos (HAIR JR. et al., 2005). Na tabela seguinte, observa-se que muitos construtos apresentaram valores superiores a $70 \%$ da variância média explicada, o que demonstra que os indicadores utilizados conseguiram medir os construtos latentes com êxito (FORNELL; LACKER, 1981; CHIN, 2010). 
Tabela 2: Variância Extraída, Confiabilidade e Consistência dos construtos

\begin{tabular}{cccc}
\hline Dimensões & $\begin{array}{c}\text { Variância Média } \\
\text { Extraída dos construtos }\end{array}$ & $\begin{array}{c}\text { Confiabilidade } \\
\text { Composta }\end{array}$ & $\begin{array}{c}\text { Consis tência Inte rna } \\
\text { (Alfa de Cronbach) }\end{array}$ \\
\hline Custos & 0,74 & 0,85 & 0,66 \\
Disponibilidade & 0,59 & 0,88 & 0,82 \\
Hábito & 0,75 & 0,90 & 0,84 \\
Rede Social & 0,70 & 0,87 & 0,79 \\
Utilidade Percebida & 0,67 & 0,90 & 0,83 \\
Uso de Telefonia IP & 0,75 & 0,86 & 0,76 \\
\hline
\end{tabular}

Quanto à confiabilidade e consistência interna, observa-se que os resultados do teste de Alfa de Cronbach indicam a maioria dos valores superiores, ou próximos, a 0,70 e são considerados satisfatórios (CRONBACH, 1951).

De acordo com a Tabela 3, observa-se que as raízes quadradas das variâncias médias extraídas dos construtos (destacadas em negrito nas duas tabelas seguintes) apresentam valores maiores que a correlação entre as variáveis latentes e, portanto, indicam que há validade discriminante entre os construtos (FORNELL; LACKER, 1981).

Tabela 3: Matriz de correlações dos construtos e raiz quadrada de AVE

\begin{tabular}{ccccccc}
\hline Dimensões & Custos & Disponibilidade & Hábito & Rede Social & $\begin{array}{c}\text { Utilidade } \\
\text { Percebida }\end{array}$ & $\begin{array}{c}\text { Uso de } \\
\text { Telefonia IP }\end{array}$ \\
\hline Custos & $\mathbf{0 , 8 6}$ & & & & & \\
Disponibilidade & 0,47 & $\mathbf{0 , 7 7}$ & & & \\
Hábito & 0,58 & 0,57 & $\mathbf{0 , 8 7}$ & & & \\
Rede Social & 0,39 & 0,54 & 0,65 & $\mathbf{0 , 8 4}$ & & \\
Utilidade Percebida & 0,61 & 0,45 & 0,62 & 0,63 & $\mathbf{0 , 8 2}$ & \\
Uso de Telefonia IP & 0,50 & 0,55 & 0,73 & 0,50 & 0,52 & $\mathbf{0 , 8 7}$ \\
\hline
\end{tabular}


Para a análise da significância dos indicadores, serão observados os valores calculados pela técnica bootstrapping (EFRON; TIBSHIRANI, 1998), parametrizada com cinco mil subamostras com reposição para 313 casos, o que é considerado adequado para pesquisas científicas (CHIN, 2010). A tabela seguinte apresenta os valores dos coeficientes entre os construtos e as respectivas estatísticas $t$ de Student também estimados pela técnica bootstrapping. Observa-se que a maioria dos coeficientes dos relacionamentos obteve significância superior a 95\% e foram considerados significantes (EFRON; TIBSHIRANI, 1998).

Tabela 4: Coeficientes do modelo estrutural

\begin{tabular}{ccccc}
\hline Relacionamento entre Dimensões & Média & $\begin{array}{c}\text { Desvio } \\
\text { Padrão }\end{array}$ & Estatística T & p-valor \\
\hline CUSTOS $\rightarrow$ HÁBITO & 0,22 & 0,05 & 3,97 & 0,00 \\
CUSTOS $\rightarrow$ UTILIDADE & 0,46 & 0,05 & 9,94 & 0,00 \\
DISPONIBILIDADE $\rightarrow$ HÁBITO & 0,18 & 0,05 & 3,38 & 0,00 \\
DISPONIBILIDADE $\rightarrow$ UTILIDADE & 0,08 & 0,05 & 1,54 & 0,13 \\
HÁBITO $\rightarrow$ USO & 0,67 & 0,04 & 15,04 & 0,00 \\
REDE SOCIAL $\rightarrow$ HÁBITO & 0,35 & 0,04 & 8,25 & 0,00 \\
REDE SOCIAL $\rightarrow$ UTILIDADE & 0,28 & 0,05 & 5,93 & 0,00 \\
UTILIDADE $\rightarrow$ HÁBITO & 0,24 & 0,05 & 4,49 & 0,00 \\
UTILIDADE $\rightarrow$ USO & 0,11 & 0,04 & 2,33 & 0,02 \\
\hline
\end{tabular}

Obtidos os resultados, ilustra-se o modelo de pesquisa com as cargas fatoriais e significâncias, além dos valores de poder de explicação dos construtos. 
Figura 2: Sintese de resultados do modelo estrutural

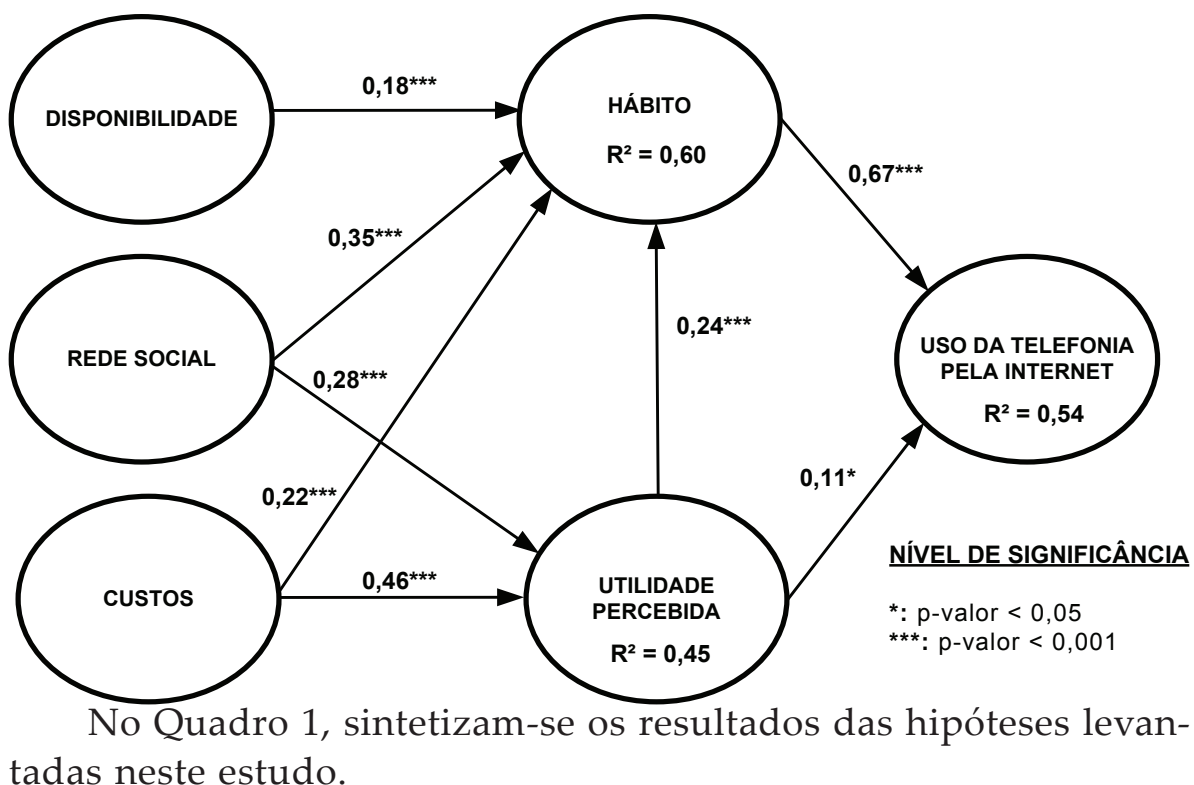

\section{Quadro 1: Resultados dos testes de Hipóteses}

\begin{tabular}{|clll|}
\hline Hipótese & \multicolumn{1}{c}{ Conteúdo } & Resultado \\
\hline H1 & $\begin{array}{l}\text { O hábito influencia positivamente o comportamento de uso da } \\
\text { telefonia pela Internet. }\end{array}$ & Confirmada \\
H2 & $\begin{array}{l}\text { A utilidade percebida influencia positivamente o comportamento de } \\
\text { uso da telefonia pela Internet. }\end{array}$ & Confirmada \\
H3 & $\begin{array}{l}\text { A utilidade percebida influencia positivamente o hábito de uso da } \\
\text { telefonia pela Internet. }\end{array}$ & Confirmada \\
H4 & $\begin{array}{l}\text { A disponibilidade influencia positivamente o hábito de uso da telefonia } \\
\text { pela Internet. }\end{array}$ & Confirmada \\
H5 & $\begin{array}{l}\text { A disponibilidade influencia positivamente a utilidade percebida da } \\
\text { telefonia pela Internet. }\end{array}$ & Não confirmada \\
H6 & $\begin{array}{l}\text { A rede social influencia positivamente a utilidade percebida da } \\
\text { telefonia pela Internet }\end{array}$ & Confirmada \\
H7 & $\begin{array}{l}\text { A rede social influencia positivamente o hábito de uso da telefonia } \\
\text { pela Internet }\end{array}$ & Confirmada \\
H8 & $\begin{array}{l}\text { Os custos percebidos influenciam positivamente a utilidade percebida } \\
\text { da telefonia pela Internet }\end{array}$ & $\begin{array}{l}\text { Os custos percebidos influenciam positivamente o hábito de uso da } \\
\text { telefonia pela Internet }\end{array}$ & Confirmada \\
\hline
\end{tabular}




\section{CONSIDERAÇõES FINAIS}

Esta pesquisa contemplou a análise de cinco construtos como influências à adoção pessoal da tecnologia VoIP. Neste caso, características individuais, influência da rede social do usuário, custos percebidos e disponibilidade foram incluídos neste estudo por envolver as decisões de uso e esclarecer o processo de adoção individual desse artefato tecnológico.

Embora a literatura de adoção de Tecnologia de Informação aborde diversos aspectos inerentes ao processo de escolha dos usuários ao uso das aplicações tecnológicas, não foram encontrados estudos que explorassem, simultaneamente, os efeitos resultantes das percepções inclusas no modelo de pesquisa concebido.

Para Cohen (1977), resultados de estudos que obtenham poder de explicação da variável dependente acima de 50\% são considerados altos, o que mostra que a seleção dos construtos antecedentes inclusos no modelo de pesquisa estudado foi satisfatória.

De acordo com os resultados, verifica-se que a adoção de uma tecnologia de comunicação pode não obedecer a uma lógica exclusiva das percepções utilitárias dos usuários. Evidenciou-se que o hábito exerce maior influência ao uso dessa tecnologia do que a percepção sobre a utilidade percebida dessa tecnologia de comunicação, ainda que um dos seus principais atrativos seja a possibilidade de comunicação telefônica sem custo tarifário, comparado com outros fatores exógenos, o que denota a sua relevância ao estudo de adoção de tecnologias.

Como os usuários dispõem de duas tecnologias de telefonia implantadas há décadas anteriores à popularização da tecnologia pela internet e estão disseminadas entre a população em número superior à comunicação pela internet, se entende que o hábito de uso das tecnologias tradicionais de telefonia formados entre eles se reflete como uma barreira a ser superada à adoção das tecnologias de telefonia pela internet.

Já a rede social de contatos do usuário é o principal fator de influência à formação do hábito de uso da tecnologia VoIP. Assim, a percepção sobre o volume, proximidade e importância dos contatos conectados a esse tipo de tecnologia corresponde, positivamente, à formação do hábito de uso dessa tecnologia. 
Entende-se que o usuário que dispõe de uma rede de contatos significativa ao seu cotidiano, pessoal ou profissional, tende a utilizar os sistemas de telefonia pela internet com maior frequência, pois visualiza, de forma subjetiva, certa probabilidade de êxito ao estabelecer uma comunicação com a sua rede de contatos.

No entanto, alguns aplicativos de telefonia pela internet permitem a ausência virtual para recebimento de chamadas pela rede social de contatos do usuário. Dessa forma, é possível que a maioria dos respondentes não permaneça disponível para a comunicação nesse tipo de tecnologia e reduza a percepção de conectividade dessa tecnologia entre os usuários e prejudique o uso habitual da aplicação.

Nesse sentido, infere-se que o aplicativo WhatsApp ${ }^{\circledR}$ que não permite que o usuário bloqueie o recebimento de chamadas telefônicas deve superar o número de chamadas telefônicas do seu principal concorrente Skype ${ }^{\circledR}$. Entende-se que a incerteza de sucesso de comunicação entre usuários fará que tentativas de ligação ocorram de forma mais frequente do que sistemas que acusam a disponibilidade virtual dos contatos.

Vale lembrar que essa incerteza sobre a efetividade de comunicação entre os contatos já ocorre com as tecnologias de comunicação tradicionais e não impediu que essas tecnologias atraíssem usuários ao longo dos anos. Assim, presume-se que a exclusão da funcionalidade de bloqueio de chamadas em aplicativos de telefonia também deve favorecer a formação do uso habitual da tecnologia analisada, entre outras razões.

Também se destaca o papel da disponibilidade da aplicação à formação do hábito de uso da telefonia VoIP, o que denota o relevante papel da infraestrutura tecnológica acessível ao usuário para que existam condições de adoção efetiva da aplicação. Assim, infere-se que a maciça utilização de sistemas VoIP será promovida pela comodidade que os smartphones com acesso à internet proporcionam aos usuários.

Postergar a oferta de acesso aos usuários a uma infraestrutura pública de redes de dados adequada para o tráfego de telefonia é uma ação que tende a dificultar a formação do hábito de uso pela dificuldade de conexão às redes de dados. Assim, políticas públicas 
que alavanquem a acessibilidade dos meios de comunicação e disseminem o acesso da população à internet com velocidade adequada de tráfego de dados possibilita que a população usufrua de tecnologias de comunicação mais sofisticadas, além de outros benefícios.

Presume-se que a baixa velocidade de internet móvel também dificulte a utilização de sistemas de telefonia VoIP. De uma forma indireta, infere-se que a baixa velocidade de internet auxilia as operadoras de telefonia tradicionais a influenciar os usuários a empregar suas redes convencionais. Provavelmente, a baixa velocidade de acesso móvel à internet deve afetar a facilidade de uso pelo desconforto ao usuário e respectiva perda de qualidade da comunicação. Assim, entende-se que a disponibilidade de redes de comunicação móveis rápidas, além de terminais de comunicação sofisticados e acessíveis promove o uso da tecnologia VoIP na população.

Além disso, observa-se que as redes de telefonia tradicionais apresentam a vantagem de possuir a integração a outras redes de telefonia de operadoras diferentes, ao contrário da tecnologia VoIP. Além da telefonia tradicional, que permite essa interligação entre redes de telefonia distintas, outros meios de comunicação também possuem interligação dos contatos de uma rede de comunicação, independentemente da aplicação de comunicação utilizada, como os e-mails.

Em outras palavras, estima-se que embora exista a disponibilidade de diferentes sistemas VoIP para uso gratuito aos usuários, essa diversidade de sistemas pode prejudicar a percepção individual de conectividade para esse tipo de tecnologia, dada a ausência de interligação entre redes distintas dos sistemas VoIP. Nesse sentido, efeitos de externalidade de rede podem ser manifestados nesse tipo de tecnologia.

Vislumbra-se uma oportunidade de desenvolvimento de um sistema central integrador de sistemas de comunicação VoIP, que permita a visualização de conexão entre outros sistemas de comunicação e favoreça a adoção desse tipo de tecnologia, independentemente da plataforma.

A inserção do construto Custos Percebidos no modelo de pesquisa também mostrou-se satisfatória aos objetivos do trabalho: a validação de seus resultados demonstrou que o benefício de tarifas 
gratuitas para comunicação entre usuários pelo sistema de telefonia pela internet é um benefício que promove o uso e causa influência à formação do uso habitual da tecnologia.

No entanto, interpreta-se que a eliminação total dos custos de uso de determinada aplicação de comunicação pode não ser percebida como a principal justificativa, à adoção individual de determinada tecnologia, visto que outros fatores analisados também se mostraram influentes ao uso da aplicação.

A elaboração de teorias e estudos sobre os fatores antecedentes, fenômenos e processos de adoção de tecnologias é importante para o desenvolvimento de inovações e sistemas que dependem da aceitação dos usuários para o aprimoramento das tecnologias atuais, entre outras justificativas.

Sob o ponto de vista dos interessados em promover o uso de tecnologias inovadoras de comunicação, este estudo contribui na exposição de direções que podem promover a maior adoção desses sistemas.

Entende-se como uma limitação da pesquisa a análise de adoção da TI com um grupo homogêneo de respondentes sobre uma única tarefa em determinado momento do tempo. Essa limitação da amostragem pode levantar questionamentos acerca da generalização das conclusões a respeito de uma população diversa.

Também podem ocorrer erros de medida por meio de imprecisão na mensuração dos valores reais das respostas, ausência de outros construtos de percepção associados no modelo de pesquisa e não pode se afirmar que a significância e valores dos testes realizados neste estudo sejam constantes dadas as diferenças de cenários de utilização, acessibilidade e desenvolvimento das tecnologias futuras.

Como o uso da telefonia pela internet é disseminado globalmente, as conclusões deste estudo de adoção dessa tecnologia podem não se restringir somente aos usuários brasileiros. Assim, a popularização mundial dessa tecnologia possibilita a replicação deste estudo em outros países, além da inserção de outros fatores antecedentes à adoção dessa tecnologia. 


\section{REFERÊNCIAS}

BHATTACHERJEE, A. Understanding information systems continuance: An expectation-confirmation model. MIS Quarterly [S.I.], v. 25, n. 3, p. 351-370, 2001.

BROWN, S. A.; VENKATESH, V. Model of adoption of technology in households: A baseline model test and extension incorporating household life cycle. MIS Quarterly [S.I.], v. 29, n. 3, p. 399-426, 2005.

BROWN, S. A.; VENKATESH, V.; GOYAL, S. Expectation confirmation in information systems research: A test of six competing models. MIS Quarterly [S.I.], v. 38, n. 3, p. 729-A9, 2014.

CECERE, G. Voip diffusion among new entrants: A path dependent process. Industry \& Innovation [S.I.], v. 16, n. 2, p. 219-245, 2009.

CHIN, W. W. The partial least squares approach to structural equation modeling. In:

CHIN, W. W. How to write and report pls analyses. In: VINZI, V. E. et al. (Org.). Handbook of partial least squares: Concepts, methods and applications. Berlin: Springer, 2010, p. 655-690.

COHEN, J. Statistical power analysis for the behavioral sciences. Revised edition. New York: Academic Press, Inc., 1977.

CRONBACH, J. L. Coefficient alpha and the internal structure of tests. Psychometrika [S.I.], v. 16, n. 3, p. 297-334, 1951.

DANTU, R.; FAHMY, S.; SCHULZRINNE, H.; CANGUSSU, J. Issues and challenges in securing voip. Computers and Security [S.I.], v. 28, n. 8, p. 743-753, 2009.

DAVIS, F. D. Perceived usefulness, perceived ease of use, and user acceptance of information technology. MIS Quarterly [S.I.], v. 13, n. 3, p. 319-340, 1989.

DAVIS, F. D.; BAGOZZI, R. P.; WARSHAW, P. R. User acceptance of computer technology: A comparison of two theoretical models. Management Science [S.I.], v. 35, n. 8, p. 982-1.003, 1989.

DE BIJL, P. W. J.; PEITZ, M. Access regulation and the adoption of voip. Journal of Regulatory Economics [S.I.], v. 35, n. 2, p. 111-134, 2009.

EFRON, B.; TIBSHIRANI, R. J. An introduction to the bootstrap. Chapman \& Hall / CRC Press, 1998.

FORNELL, C.; LACKER, D. F. Evaluating structural equation models with unobservable variables and measurement error. Journal of Marketing Research (JMR) [S.I.], v. 18, p. 39-50, 1981.

GLASS, R.; LI, S. Social influence and instant messaging adoption. Journal of Computer Information Systems [S.I.], v. 51, n. 2, p. 24-30, 2010.

GUINEA, A. O.; MARKUS, M. L. Why break the habit of a lifetime? Rethinking the roles of intention, habit, and emotion in continuing information technology use. MIS Quarterly [S.I.], v. 33, n. 3, p. 433-444, 2009.

GUPTA, S.; KIM, H.-W. Value-driven internet shopping: The mental accounting theory perspective. Psychology \& Marketing [S.I.], v. 27, n. 1, p. 13-35, 2010. 
HAIR JR., J. F.; BABIN, B.; MONEY, A. H.; SAMOUEL, P. Fundamentos de métodos de pesquisa em administração. Porto Alegre: Bookman, 2005.

HU, P. J.-H.; BROWN, S. A.; THONG, J. Y. L.; CHAN, F. K. Y.; TAM, K. Y. Determinants of service quality and continuance intention of online services: The case of etax. Journal of the American Society for Information Science \& Technology [S.I.], v. 60, n. 2, p. 292-306, 2009.

IDG. Um em cada quatro americanos já fez ligações telefônicas via internet, 2011. Disponível em: <http://idgnow.uol.com.br/internet/2011/05/30/um-em-cada-quatro-americanos-ja-fez-ligacoes-telefonicas-via-internet/>. Acesso em: 11.08.2011.

KERAMATI, A.; TAEB, R.; LARIJANI, A. M.; MOJIR, N. A combinative model of behavioural and technical factors affecting 'mobile'-payment services adoption: An empirical study. Service Industries Journal [S.I.], v. 32, n. 9, p. 1.489-1.504, 2012.

KIM, G. S.; PARK, S.-B.; OH, J. An examination of factors influencing consumer adoption of short message service (sms). Psychology \& Marketing [S.I.], v. 25, n. 8, p. 769-786, 2008.

KIM, S. S. The integrative framework of technology use: An extension and test. MIS Quarterly [S.I.], v. 33, n. 3, p. 513-537, 2009.

KIM, S. S.; MALHOTRA, N. K. A longitudinal model of continued is use: An integrative view of four mechanisms underlying postadoption phenomena. Management Science [S.I.], v. 51, n. 5, p. 741-755, 2005.

KWAK, J. H.; LEE, B. G. How the Rapid Development of VoIP Technologies Affects Consumer Welfare and the Market. Global Economic Review, v. 41, n. 3, p. 243-257, 2012.

LIMAYEM, M.; HIRT, S. G.; CHEUNG, C. M. K. How habit limits the predictive power of intention: The case of information systems continuance. MIS Quarterly [S.I.], v. 31, n. 4, p. 705-737, 2007.

LOHMOLLER, J. Latent variable path modeling with partial least squares. Heidelberger: Physica-Verlag, 1989.

MARKUS, M. L. Toward a 'critical mass' theory of interactive media: Universal access, interdependence and diffusion. Communication Research [S.I.], v. 14, p. 491-511, 1987.

PESSEMIER, T. et al. Quality assessment and usage behavior of a mobile voice-over-IP service. Telecommunication Systems, v. 61, n. 3, p. 417-432, 2016.

POLITES, G. L; KARAHANNA, E. Shackled to the status quo: The inhibiting effects of incumbent system habit, switching costs, and inertia on new system acceptance. MIS Quarterly [S.I.], v. 36, n. 1, p. 21-A13, 2012.

RINGLE, C. M.; WENDE, S.; WILL, A. Smartpls. Hamburg, Germany, 2005.

ROGERS, E.M. Diffusion of innovations, Fourth Edition ed. New York: Free Press, 1995.

SHIN, D. H. What makes consumers use VoIP over mobile phones? Free riding or consumerization of new service. Telecommunications Policy, v. 36, n.4, p. 311-323. doi: 10.1016/j. telpol.2012.01.004, 2012.

TELECO. Celulares por região smp/smc/estado (mar. 12), 2012. Disponível em:<http://www. teleco.com.br/nceluf.asp>. Acesso em: 24.04.2012. 
TOBIN, P. K. J.; BIDOLI, M. Factors affecting the adoption of voice over internet protocol (voip) and other converged ip services in south africa. South African Journal of Business Management [S.I.], v. 37, n. 1, p. 31-40, 2006.

VAN SLYKE, C.; ILIE, V.; STAFFORD, T. Perceived critical mass and the adoption of a communication technology. European Journal of Information Systems [S.I.], v. 16, p. 270-283, 2007.

VENKATESH, V.; MORRIS, M. G.; DAVIS, G. B.; DAVIS, F. D. User acceptance of information technology: Toward a unified view. MIS Quarterly [S.I.], v. 27, n. 3, p. 425-478, 2003.

VENKATESH, V.; THONG, J. Y. L.; XU, X. Consumer acceptance and use of information technology: extending the unified theory of acceptance and use of technology. MIS Quarterly, v. 36, n. 1, p. 157-178, 2012.

VINZI, V. E.; CHIN, W. W.; HENSELER, J.; WANG, H. Handbook of partial least squares: Concepts, methods and applications. London: Springer Heidelberg Dordrecht, 2010.

XIN, L.; GURUNG, A.; SHIM, J. P. Understanding the determinants of user acceptance of enterprise instant messaging: An empirical study. Journal of Organizational Computing \& Electronic Commerce [S.I.], v. 20, n. 2, p. 155-181, 2010.

Recebido em: 6-10-2017

Aprovado em: 6-4-2018

Avaliado pelo sistema double blind review.

Editor: Coordenação do PPGA/UMESP

Disponível em http://mjs.metodista.br/index.php/roc 